\title{
TESTES FÍSICOS: UMA REVISÃO SOBRE AS AVALIAÇÕES PRÁtICAS E ACESSÍVEIS DE CAPACIDADE AERÓBIA, ANAERÓBIA E AGILIDADE NO FUTEBOL DE BASE
}

\section{ARTIGO DE REVISÃO}

SILVA, Matheus Luís da ${ }^{1}$

SILVA, Matheus Luís da. Testes físicos: uma revisão sobre as avaliações práticas e acessíveis de capacidade aeróbia, anaeróbia e agilidade no futebol de base. Revista Científica Multidisciplinar Núcleo do Conhecimento. Ano. 06, Ed. 12, Vol. 01, pp. 22-32. Dezembro de 2021. ISSN: 2448-0959, Link de acesso: https://www.nucleodoconhecimento.com.br/educacao-fisica/avaliacoes-praticas, DOI: 10.32749/nucleodoconhecimento.com.br/educacao-fisica/avaliacoes-praticas

\section{RESUMO}

Visando a necessidade de monitorar as variáveis mensuráveis dos atletas de futebol, os testes físicos se fazem necessários. Avaliar, analisar e utilizar os dados, são funções dos preparadores físicos e fisiologistas, dando um melhor direcionamento dos treinamentos otimizando os resultados. Dentro dos possíveis dados que possam ser mensurados, nas categorias de base, as avaliações da capacidade anaeróbia, aeróbia e a agilidade são de extrema importância. Existem diversas maneiras de se avaliar essas variáveis, porém os testes devem ser os mais fidedignos e próximos da modalidade possíveis. Tendo em vista este contexto, o presente artigo tem como questão norteadora: quais são os testes mais indicados para avaliar capacidade aeróbia, anaeróbia e agilidade? Para responder a esta questão, objetivou-se realizar uma revisão bibliográfica nas plataformas Scielo, PubMed e google acadêmico para apresentar os testes mais indicados para avaliar as variáveis de capacidades anaeróbia, aeróbia e agilidade. Dentro da literatura foram encontrados diversos testes e protocolos, sendo os testes Rast, Yoyo test

\footnotetext{
${ }^{1}$ Pós-graduando Treinamento desportivo alto rendimento - CEFIT.
}

RC: 102512

Link de acesso: https://www.nucleodoconhecimento.com.br/educacaofisica/avaliacoes-praticas 
recovery e llinois sucessivamente os mais indicados para se avaliar essas capacidades.

Palavras-chave: Avaliação física, futebol, atletas.

\section{INTRODUÇÃO}

O futebol é um esporte de característica intermitente, onde as demandas físicas durante a partida se diferem. (IMPELLIZERI, 2008). Com predominância do metabolismo aeróbio durante o jogo, cerca de $90 \%$ da energia vem dessa via metabólica, porém, em momentos cruciais a demanda energética se dá por movimentos onde a predominância são das vias anaeróbias. (BANGSBO, 1994).

O jogador dentro de uma partida realiza diversos movimentos, como, chutar, correr, cabecear, driblar, entre outros. Diversas variáveis são encontradas em um atleta denominado completo. Assim as mais importantes encontradas são a velocidade, resistência, força, agilidade e a flexibilidade. (IMPELLIZERI, 2008).

O controle e avaliação do processo de treino são ferramentas fundamentais para a prescrição dentro de um planejamento, os preparadores físicos e fisiologistas necessitam de dados para montagem dos treinamentos, nesse cenário entra as avaliações físicas, para diagnosticar a atual forma do jogador. Os valores podem ser utilizados para o controle da carga do atleta, valores de referência de reabilitação e prevenção, além de auxiliar os profissionais a realizarem ajustes no treino. (FONSECA, 2007). Existem uma ampla possibilidade de protocolos de testes que podem ser utilizados, porém deve se ter um certo cuidado ao escolher. Alguns parâmetros devem ser observados, devido a essa proximidade com a modalidade e assim as avaliações físicas foram ao longo do tempo se tornando cada vez mais específicas.

Como pilares das baterias de testes físicos, as avaliações de capacidade anaeróbia, aeróbia e agilidade são de extrema importância, mas quais são os testes mais indicados para avaliar essas variáveis? Existem diversos testes para se avaliar,

RC: 102512

Link de acesso: https://www.nucleodoconhecimento.com.br/educacaofisica/avaliacoes-praticas 
portanto escolher os testes mais indicados e mais fidedignos se fazem necessários para aproximar os resultados das demandas que o atleta necessita em campo, com base nisso, a escolha dos testes dessas variáveis deve ser feita de maneira bem minuciosa. O presente estudo, coletou trabalhos, encontrados nas plataformas Scielo, PubMed e google acadêmico, selecionando assim os testes mais indicados na literatura para responder à questão norteadora.

$\mathrm{Na}$ categoria de base, os jovens atletas demandam de cuidados necessários importantes, além de conhecer indicadores do atleta a avaliação pode corroborar na detecção de talentos, portanto, observando a necessidade da aplicação de testes físicos em atletas de futebol, esse presente artigo tem como objetivo com base na literatura prepor sobre as avaliações práticas e acessíveis de capacidade aeróbia, anaeróbia e agilidade no futebol de base.

Esta pesquisa tem como metodologia uma revisão da literatura. Sendo realizada a busca em livros e pesquisas sites como Pubmed, Scielo e google acadêmico.

\section{CAPACIDADE AERÓBIA}

O consumo máximo de oxigênio é uma variável utilizada para a mensuração da aptidão aeróbia representada pelo VO2MAX, portanto é o parâmetro para avaliar a condição aeróbia do indivíduo. É uma variável extremamente necessário para o atleta, permitindo uma constância e ritmo durante uma partida de futebol. (PEREIRA et al., 2016, MCARDLE; KATCH, 2001).

Esse consumo de oxigênio depende muito do nível de treinamento do atleta. Quanto maior o VO2MAX, maior é sua capacidade respiratória. Seus valores podem ser expressos tanto em valores absolutos quanto em valores relativos. Dentro de uma partida de futebol, essa condição é responsável por $90 \%$ da condição do atleta, porém não determinante.

Dentro da literatura podemos encontrar diversos protocolos de avaliação dessa variável. Cezar e Fantinel (2011) realizaram um estudo comparando os métodos de

RC: 102512

Link de acesso: https://www.nucleodoconhecimento.com.br/educacaofisica/avaliacoes-praticas 
avaliações indiretas de Cooper e Yoyo test level 1, com atletas da categoria sub 15 masculinos de uma equipe de futebol, os testes foram aplicados com diferença de 2 dias. Os resultados encontrados foram que os testes têm uma boa correlação para mensuração da aptidão aeróbia dessa categoria.

Nunes (2021) realizou em seu estudo, comparando avaliação da aptidão aeróbia com jogadores das categorias sub 13,15, 17 e sub 20. O teste utilizado foi o Yoyo test e destacado a característica intermitente do teste, aproximando ao máximo os movimentos executados no futebol, sendo o melhor aplicado para avaliação dessa variável. Silva (2011) realizou uma pesquisa em relação a testes de campo no futebol, dentre eles foi citado Shuttlerun 20m, Yoyo test recovery 11 e o T-Car, obtendo o resultado fidedignos entre os dois últimos testes no futebol. Lizana et al. avaliou atletas da categoria sub 20 de um clube de futebol, para mensurar a aptidão aeróbia de maneira indireta, foram comparados os testes de troca de gases na esteira e o Yoyo test 11. Os resultados foram de correlação entre os testes.

Tabela 1 - Estudos relacionando método indireto de avaliação aeróbia e seus resultados.

\begin{tabular}{|c|c|c|c|c|}
\hline Autor & Público & $\mathrm{N}^{\circ}$ & Teste & Conclusão \\
\hline $\begin{array}{l}\text { Cezar e } \\
\text { Fantiel } \\
(2011)\end{array}$ & Cat. sub 15 & 8 & $\begin{array}{l}\text { Cooper e Yoyo } \\
\text { test } L 1\end{array}$ & $\begin{array}{l}\text { Correlação entre os } \\
\text { resultados }\end{array}$ \\
\hline $\begin{array}{l}\text { Nunes } \\
\text { (2021) }\end{array}$ & $\begin{array}{l}\text { Cat. sub } 13,15, \\
17 \text { e } 20\end{array}$ & * & Yoyo Test & Protocolo fidedigno yoyo test \\
\hline $\begin{array}{l}\text { Silva } \\
\text { (2011) }\end{array}$ & $\begin{array}{l}\text { Não } \\
\text { especificado }\end{array}$ & 18 & $\begin{array}{l}\text { Shuttlerun } 20 m \text {, } \\
\text { Yoyo Test I1 e } T- \\
\text { Car }\end{array}$ & $\begin{array}{l}\text { Os testes T-car e Yoyo test } \\
\text { foram os protocolos mais } \\
\text { indicados para o futebol }\end{array}$ \\
\hline $\begin{array}{l}\text { Lizana et } \\
\text { al. (2011) }\end{array}$ & Sub 20 & 24 & $\begin{array}{l}\text { Esteira (troca de } \\
\text { gases) e yoyo }\end{array}$ & Correlação entre os testes \\
\hline
\end{tabular}


test $/ 1$

Fonte: Elaborado pelo autor

Dentro dos testes indiretos para mensuração da aptidão aeróbia, o yoyo test atualmente, é o teste mais utilizado para mensuração dessa variável, devido as características que se aproximam do futebol.

\section{CAPACIDADE ANAERÓBIA}

As ações intensas dentro de uma partida de futebol, são movimentos onde pode ser considerado decisivo no resultado da partida. As ações anaeróbias na modalidade são expressas em sprints máximos ou submáximos, corridas intermitentes alta intensidade com alguns intervalos. (BANGSBO, 2007).

Os protocolos de avaliações indiretas para essa variável envolvem componentes de velocidade, simples e complexos.

Para avaliação da potência anaeróbia máxima, o teste de 10 metros é um exemplo de teste de fácil aplicação e baixo custo. $\mathrm{O}$ atleta realiza um Sprint de 10 metros no menor tempo possível. $O$ teste deve ser realizado em campo com a utilização de chuteira, aproximando ao máximo o teste com a especificidade do jogo. (SVENSSON, 2007).

\section{RAST (RUNNING-BASED ANAEROBIC SPRINT TEST)}

Um teste muito encontrado dentro da literatura para mensuração da resistência anaeróbia é o RAST. Esse teste é altamente acessível, onde é possível detectar a potência máxima, potência média e potência mínima, além do índice de fadiga. Esse método de avaliação necessita de uma alta demanda do metabolismo anaeróbia, por isso um aquecimento prévio se faz necessário.

RC: 102512

Link de acesso: https://www.nucleodoconhecimento.com.br/educacaofisica/avaliacoes-praticas 
O teste consiste em 6 sprints de 35 metros com o intervalo de 10 segundos. Deve ser registrado o tempo de cada sprint. Alguns estudos comprovam a eficácia desse teste. Souza (2012) em seu estudo comparou o teste de RAST em jogadores juniores com outros testes de agilidade e força explosiva. Chegou-se à conclusão de que o RAST não está associado a força, tão pouco agilidade, porém é um excelente teste para mensuração da capacidade aeróbia.

Kalva-Filho (2013) comparou os resultados de potência anaeróbia pelo teste de RAST em diferentes condições de calçados e superfícies, o resultado obtido foi evidenciado que o tipo de superfície influência no resultado diretamente da avaliação.

Para avaliar a melhor distribuição da carga de treinamento dos atletas sub15 e sub17 de uma equipe de futebol, Figueiredo (2020) utilizou o protocolo do RAST para mensuração da capacidade anaeróbia por conta de sua fidedignidade, obtendo os dados de potência máxima, potência média, potência mínima e índice de fadiga.

Dal Pupo (2010) a fim de avaliar os níveis de potência muscular de atletas da categoria sub 20 de um time de futebol, utilizou o teste de RAST para avaliar a capacidade de sprints repetidos dos atletas, sendo uma das boas alternativas devido à proximidade com o futebol.

Gagliardi (2015) realizou uma revisão literária buscando encontrar a melhor maneira de mensurar a potência anaeróbia de atletas de futebol, foi encontrado que RAST apresenta ser o teste mais específico para o futebol para mensuração da potência anaeróbia.

Tabela 2 - Estudos relacionado o RAST e sua fidedignidade nos testes de capacidade anaeróbia.

\begin{tabular}{|l|l|l|l|l|}
\hline Autor & Público & N$^{\circ}$ & Sexo & Conclusão \\
\hline Kalva-Filho & Sub 17 & 8 & Masc & O tipo da superfície e calçado influência
\end{tabular}

RC: 102512

Link de acesso: https://www.nucleodoconhecimento.com.br/educacaofisica/avaliacoes-praticas 
et al. (2013)

Souza et al. Juniores (2012)

no resultado do teste de Rast

16 Masc Rast é o mais indicado para avaliação da capacidade anaeróbia para atletas de futebol

Figueiredo Sub 15 e 1717 Masc Potência máxima, potência média, (2020) potência mínima e índice de fadiga

Dal Pupo et Sub 20

20 Masc Capacidade Sprints repetidos

al. (2010)

Gagliardi

Revisão de

Masc $R a$ literatura

específico para o futebol para mensuração da potência anaeróbia.

Fonte: Elaborado pelo autor.

\section{AGILIDADE}

A agilidade no futebol é uma qualidade extremamente necessária para a modalidade. É expressa em forma de ações com mudanças de direção, equilíbrio entre outras. Para avaliar a agilidade, existem inúmeros testes. (WILLIANS, 2005).

Os testes mais comuns que encontramos são ShuttleRun, o T-Test, Illinois Agility Test e o teste do quadrado. Para o futebol diversas adaptações podem ser realizadas como a utilização da bola ou a realização do teste no gramado.

Especificamente para o futebol os protocolos de Illinois, $\mathrm{T}$ teste e o teste do quadrado pode ser utilizado para avaliar atletas jovens e incrementando adaptações sempre que necessário para aproximar ao máximo o teste da realidade do jogo.

Os testes de agilidade, necessitam obedecer a alguns padrões necessários, como a tomada de decisão ou componente reativo, velocidade na mudança de direção entre outros aspectos. 
O teste de Illinois é validado por Sheppard (2006), também foi confirmado em um estudo mais recente de Oliveira (2017), sendo um teste que é altamente adaptável, que pode vir a ser realizado tanto em grama, com chuteira ou em alguns casos até utilizando o componente da bola.

Imagem 1 - Illinois test

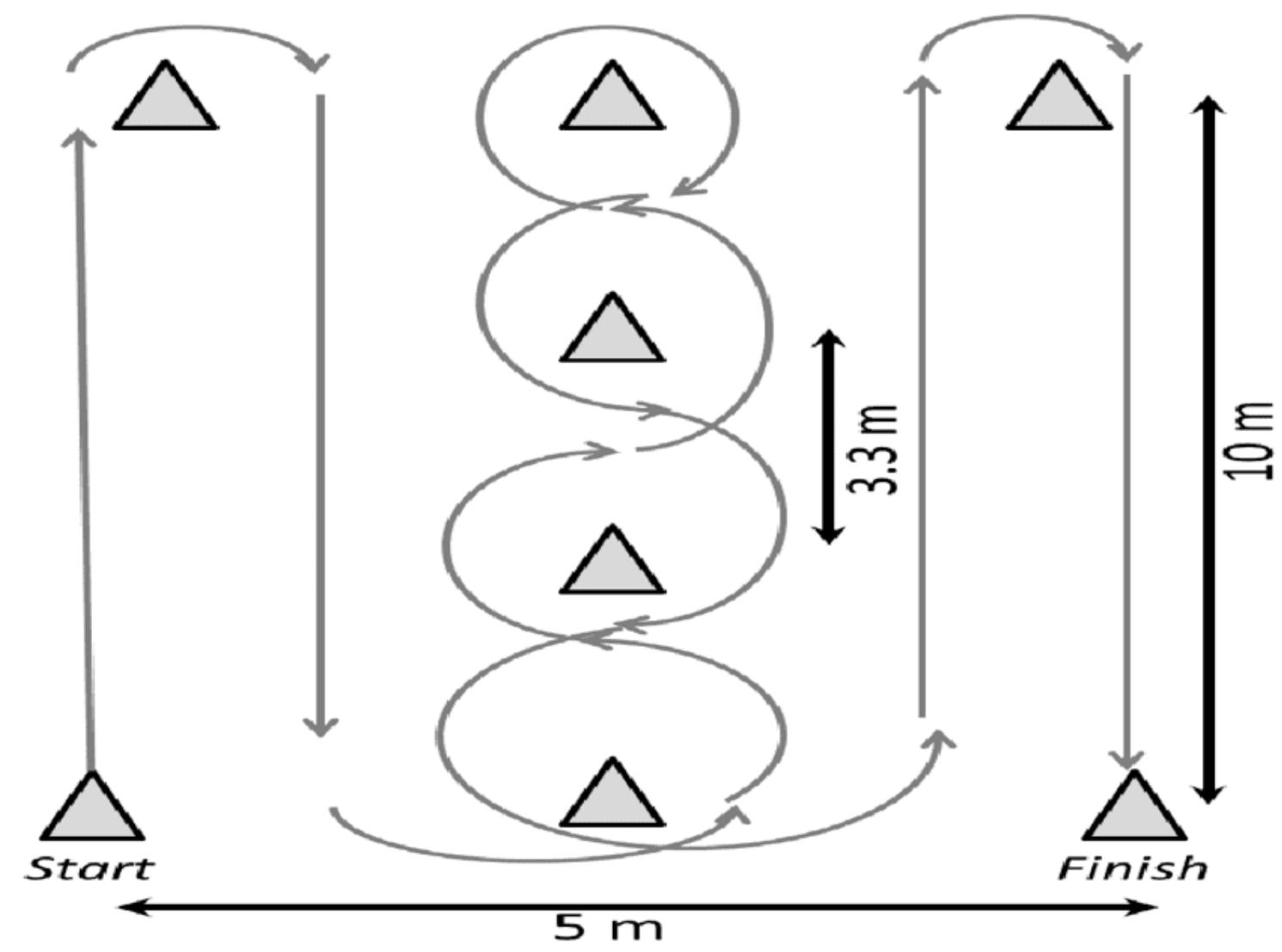

Fonte: Agility literature review: classifications, training and testing, Sheppard (2006).

Dentro dos testes apresentados, os testes de ilinois e $T$ - test se destacam na literatura, podendo ser realizados de maneira adaptada, dentro das necessidades da equipe. 


\section{CONCLUSÃO}

A avaliação física no futebol é essencial para planejar as sessões de treinamento e ter um mapeamento do atleta. Alguns protocolos podem ser totalmente acessíveis, de maneira indireta para algumas variáveis. A ideia do presente artigo se deu pela imensa quantidade de protocolos e testes para se avaliar as capacidades aeróbias, anaeróbia e agilidade no futebol da categoria de base.

Dentro das avaliações acessíveis foram encontrados diversos testes para mensurar essas variáveis, por meio dos artigos e materiais estudados, ficou evidente que os testes os mais utilizados foram para capacidade aeróbia o Yoyo test, para capacidade anaeróbia o Rast parece ser o teste mais completo; na avaliação de agilidade os testes de Illinois é que mais se destaca em relação ao futebol. Para complementar a bateria de testes, avaliações antropométricas e de força também são necessárias.

Diante disso, pode-se considerar que os testes citados acima podem ser utilizados para se avaliar os atletas da categoria de base de futebol, por ter correlação direta com a modalidade. Os testes devem se aproximar ao máximo da realidade do jogo e sempre deve se avaliar as variáveis que irá de fato ser utilizada, nunca avaliar apenas para ter os dados sem um objetivo. Pesquisas práticas são necessárias para comprovação desse estudo.

\section{REFERÊNCIAS}

BANGSBO J. Energy demands in competitive soccer. J Sports Sci. 1994;12(sup1):S5-12.

BANGSBO J, laia FM, Krustrup P. Metabolic response and fatigue in soccer. Int $\mathrm{J}$ Sports Physiol Perform. 2007;2(2):111-2 
CEZAR D. G. M.; DOS REIS, L. F. Comparação entre o teste de cooper e o yoyo endurance test 11 para predição do VO2 máximo em jogadores de futebol amador sub-15. RBFF - Revista Brasileira de Futsal e Futebol, Vol. 3. 2012. Num. 7, 5 fev.

DAL PUPO J, Almeida CMP, Detanico D, Da Silva JF,Guglielmo L. Potência muscular e capacidade de sprints repetidos em jogadores de futebol. Rev Bras Cineantropom Desempenho Hum. 2010;12(4):255-61.

FIGUEIREDO, D. H.; Matta, M. O. Impacto da distribuição das cargas de treinamento no desempenho físico de jogadores de futebol durante a pré-temporada. Revista Brasileira de Educação Física e Esporte, [S. I.], v. 34, n. 4, p. 685-697, 2020. DOI: 10.11606/issn.1981-4690.v34i4p685-697.

Disponível em: https://www.revistas.usp.br/rbefe/article/view/180275.

FONSECA ST, Ocarino JM, Silva PLP, Bricio RS, Costa CA, Wanner LL. Caracterização da performance muscular em atletas profissionais de futebol. Rev Bras MedEsporte. 2007; 13:1437.

IMPELLIZERI FM, Rampinini E, Castagna C, et al. Validity of a repeatedsprint test for football. Int J Sports Med. 2008;29(11):899-905.

KALVA-FILHO CA, Loures JP, Franco VH, Kaminagakura El, Zagatto AM, Papoti M. Comparação da potência anaeróbia mensurada pelo teste de RAST em diferentes condições de calçado e superfícies. Rev Bras Med Esp. 2013; 19:13942.

LITTLE T, Williams AG.Specificity of acceleration,maximum speed, and agility in professional soccer players. JStrength Cond Res. 2005;19(1):76-8

LIZANA, Cristian Javier Ramirez et al. Análise da potência aeróbia de futebolistas por meio de teste de campo e teste laboratorial. Rev Bras Med Esporte, São Paulo v. 20 , n. 6, p. 447-450, Dec. 2014. Available from <http://www.scielo.br/scielo.php?script=sci_arttext\&pid=S1517 
MCARDLE WD, Katch FI, Katch VL. Exercise physiology. nutrition, energy and human performance.7th ed. Guanabara Koogan; 5th ed 2011

NUNES et al. Yo-Yo Intermittent Recovery Test (Yo-Yo Test) em categorias de base. Rev Bras Futebol 2021; v. 14, n. 1, 20 - 34

PEREIRA L.N.; Monteiro A.N.; Franca J, E.G.; Barreto J.G.; Pereira R.; Machado M. Correlação entre o VO2max estimado pelo Teste de Cooper de 12 minutos e pelo Yo-Yo Endurance Test L1 em atletas de futebol. Revista Brasileira de Futebol, vol. 1, num. 1. 2008 p. 33-41.

OLIVEIRA, P. Testes físicos para avaliação da agilidade: possibilidade de adaptação ao futebol. The Brazilian Journal of Soccer Science, 2017 8, 65-75.

SHEPPARD, J. M., \& Young, W. B. (2006). Agility literature review: classifications, training and testing. Journal of sports sciences, 24(9), 919-932. https://doi.org/10.1080/02640410500457109

SILVA, J. R. Diferença nos resultados dos testes de consumo de oxigênio em atletas de futebol utilizando protocolos de análise direta e indireta. EFDeportes.com, Revista Digital. Buenos Aires. Año 16. N. 165. 2012

SOUZA V.; Pires FdO, Lima-Silva AE, Bertuzzi R. Relação entre o desempenho no Running-based Anaerobic Sprint Test (RAST) e a altura do salto vertical, salto horizontal e agilidade em futebolistas. Acta Bras Mov Hum. 2012;2(1):34-45.

SVENSSSON M, Drust B. Testing soccer players. J Sports Sci. 2005;23(6):60118

Enviado: Maio, 2021.

Aprovado: Dezembro, 2021. 